\title{
Impact of SPRINT results on hypertension guidelines: implications for "frail" elderly patients
}

\author{
Gennaro Russo ${ }^{1}$ Ilaria Liguori ${ }^{1}$ - Luisa Aran ${ }^{1}$ Giulia Bulli ${ }^{1}$ Francesco Curcio ${ }^{1}$ - Gianluigi Galizia ${ }^{2}$. \\ Gaetano Gargiulo $^{3}$ - Gianluca Testa ${ }^{1,4} \cdot$ Andrea Ungar $^{5} \cdot$ Francesco Cacciatore $^{1,6}$ - Domenico Bonaduce ${ }^{1}$. \\ Pasquale Abete ${ }^{1}$
}

Received: 22 March 2018 / Revised: 12 June 2018 / Accepted: 18 June 2018

(c) Macmillan Publishers Limited, part of Springer Nature 2018

\begin{abstract}
In the last years, guidelines for the treatment of hypertension recommended individualized blood pressure goals for geriatric population because of elderly susceptibility to adverse outcomes and higher mortality rate deriving from the excessive blood pressure lowering, especially in "frail" elderly. Recent findings from the SPRINT study, which demonstrated that intensive blood pressure lowering was associated with lower rates of cardiovascular events and mortality in both hypertensive fit and frail elderly subjects compared to standard treatment, heavily influenced the recent US guidelines. In SPRINT sub-study analysis of adults aged $\geq 75$ years, the most controversial issue appears the method of blood pressure measurement, the selection of patients and related-frailty degree that appears to be very light. Accordingly, it has been described that light frailty is related to good outcomes in older adults. SPRINT findings in "frail elderly patients" cannot be applied to the clinical practice because this condition has been clearly under-estimated. Thus, frailty status should be routinely and correctly quantified in order to identify the frailty degree and to find the best harms-benefits balance of antihypertensive drug treatment in frail older adults.
\end{abstract}

Electronic supplementary material The online version of this article (https://doi.org/10.1038/s41371-018-0086-6) contains supplementary material, which is available to authorized users.

Pasquale Abete

p.abete@unina.it

1 Department of Translational Medical Sciences, University of Naples "Federico II", Naples, Italy

2 Istituti Clinici Scientifici Maugeri- Syncope unit - UOC Cure subacute, Milan, Italy

3 Division of Internal Medicine, AOU San Giovanni di Dio e Ruggi di Aragona, Salerno, Italy

4 Department of Medicine and Health Sciences, University of Molise, Campobasso, Italy

5 Azienda Ospedaliero-Universitaria Careggi, University of Florence, Florence, Italy

6 Azienda Ospedaliera dei Colli, Monaldi Hospital, Heart Transplantation Unit, Naples, Italy

\section{Introduction}

Age-related chronic diseases are largely prevalent in frail elderly leading to polipharmacy and relative adverse outcomes as well as severe functional limitation. Hypertension, especially isolated systolic pattern, is a widespread condition in geriatric population, representing a well-established risk factor for cardiovascular disease (CVD), chronic kidney disease (CKD) and cognitive decline [1]. Based on several trials [2-4] and meta-analysis, in the last years guidelines for the treatment of hypertension recommended different blood pressure (BP) goals for geriatric population compared to young adults because of elderly susceptibility to adverse outcomes deriving from the excessive BP lowering such as falls, fractures, and cognitive impairment.

Most recently, the Systolic blood PRessure INtervention Trial (SPRINT) [5], a large prospective study that compared the effects of an intensive treatment of hypertension with a standard one, has provided data showing a better outcome in older adults treated with an aggressive antihypertensive regimen: in a sub-group analysis of subjects aged $>75$ years, the intensive treatment group had significantly lower rates of CVD and mortality compared to participants receiving 
Table 1 Different BP goals recommended by international guidelines related to hypertensive subjects cluster in pre-SPRINT era

\begin{tabular}{lllllll}
\hline BP goal & $\begin{array}{l}\text { ESH/ESC } \\
(2013)[13]\end{array}$ & $\begin{array}{l}\text { JNC-8 } \\
(2014)[14]\end{array}$ & $\begin{array}{l}\text { ASH/ISH } \\
(2014)[15]\end{array}$ & $\begin{array}{l}\text { AHA/ACC } \\
(2015)[16]\end{array}$ & $\begin{array}{l}\text { CHEP } \\
(2017)[25]\end{array}$ & $\begin{array}{l}\text { AHA/ACC } \\
(2017)[26]\end{array}$ \\
\hline Age $<60$ & $<140 / 90$ & $<140 / 90$ & $<140 / 90$ & $<140 / 90$ & $<140 / 90$ & $<130 / 80$ \\
Age $60-79$ & $<140 / 90$ & $<150 / 90$ & $<140 / 90$ & $<140 / 90$ & $<140 / 90$ & $<130 / 80$ \\
Age $>80$ & $<150 / 90$ & $<150 / 90$ & $<150 / 90$ & $<150 / 90$ & $<140 / 90$ & $<130 / 80$ \\
Diabetes & $<140 / 85$ & $<140 / 90$ & $<140 / 90$ & $<140 / 90$ & $<130 / 80$ & $<130 / 80$ \\
CKD & $<130 / 90$ & $<140 / 90$ & $<140 / 90$ & $<140 / 90$ & $<140 / 90$ & $<130 / 80$ \\
\hline
\end{tabular}

ESC European society of cardiology; ESH European society of hypertension; JNC-8 eighth joint national committee; $A S H$ American society of hypertension; ISH international society of hypertension; $A H A$ American heart association; ACC American college of cardiology; CHEP Canadian hypertension education program; $C K D$ chronic kidney disease; $B P$ blood pressure standard treatment [6]. The purpose of this review is to critically analyze SPRINT Study's results and BP goals in elderly population based on the evidence available in literature.

\section{RCT and observational study in older hypertensive subjects}

In the last twenty years, several trials have observed positive effects in treating hypertension in older adults [2-4], but no study aimed to reach a SBP target lower than $140 \mathrm{mmHg}$. On the contrary, clinical trials which achieved a SBP lower than $140 \mathrm{mmHg}$ showed no benefits in reaching this goal $[7,8]$. In the hypertension in the very elderly trial (HYVET) [4], patients aged $>80$ years with SBP $>160 \mathrm{mmHg}$ were randomized in two groups treated with placebo or indapamide alone or in association with perindopril. The results of this study showed that hypertension treatment, based on indapamide with or without perindopril aimed to achieve a target $\mathrm{BP}$ of $150 / 80 \mathrm{mmHg}$, was associated with lower mortality rate. The Japanese trial to assess optimal systolic blood pressure in elderly hypertensive patients (JATOS) [7], evaluated the effect of hypertension treatment with a SBP target $<140 \mathrm{mmHg}$ compared to a SBP target among $140-160 \mathrm{mmHg}$, showing no evidence of clinical benefit from aggressive BP lowering compared with standard BP target in elderly aged $>75$ years. The results of this study confirmed that a strict BP control may not enhance the clinical benefit in the prevention of cardiovascular events as compared with that of moderate BP control. Benetos et al. recently published the results of the PARTAGE Study, (predictive values of blood pressure and arterial stiffness in institutionalized very aged population), a multicenter, observational study investigating the relationship among mortality rate, SBP levels and number of antihypertensive drugs (AHDs) in the elderly [9]. In a sample of 1130 frail older adults aged $>80$ years, living in nursing homes, the study compared the subjects with a $\mathrm{SBP}<130 \mathrm{mmHg}$ receiving a combination of AHDs with all other participants. Their results showed that, among very old frail institutionalized subjects, the sub-group with lower SBP $(<130 \mathrm{mmHg})$, receiving an association of different AHDs, had a doubled risk of mortality [9].

In The Singapore Chinese Health Study [10], Koh et al. investigated the association between SBP categories and CVD mortality among middle-aged and elderly adults with and without CVD history. Data obtained provided evidence that SBP between 120 and $139 \mathrm{mmHg}$ values were associated with the lowest risk of CVD mortality in middle aged and elderly adults, regardless of underlying CVD. More importantly, a higher rate of events above and below this BP range were observed [10].

\section{Guidelines pre-SPRINT}

Over time, several guidelines suggested different BP targets (Table 1). In 2013, the European society of hypertension/ European society of cardiology recommended a BP target $<140 / 90 \mathrm{mmHg}$ and a SBP goal between 140 and $150 \mathrm{mmHg}$ for elderly aged $>80$ years [11]. In the same year, Eighth Joint National Committee (JNC 8) guidelines recommended a target $\mathrm{BP}<150 / 90 \mathrm{mmHg}$ in older adults without diabetes mellitus or CKD and $<140 / 90 \mathrm{mmHg}$ in the presence of these two conditions [12]. In the 2014 American society of hypertension guidelines identified the threshold for treatment at SBP values $<150 \mathrm{mmHg}$ in subjects aged $>80$ years [13]. In 2015, both "American college of cardiology foundation/American heart association (ACC/ AHA)" and "Canadian hypertension education program (CHEP)" recommended BP values $<140 / 90 \mathrm{mmHg}$, and $>150 / 90 \mathrm{mmHg}$ for octogenarians if tolerated [14].

\section{Results of the SPRINT sub-study in the elderly}

The SPRINT Study [5] compared an intensive $(\mathrm{SBP}<120 \mathrm{mmHg})$ to a standard $(\mathrm{SBP}<140 \mathrm{mmHg})$ 


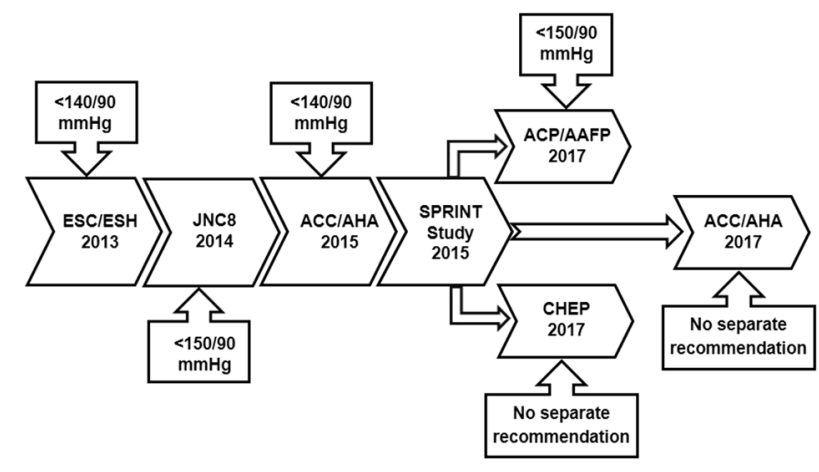

Fig. 1 Blood pressure (BP) target in hypertensive older adults recommended by guidelines from pre- to post-SPRINT era (AHA American heart association; ACC American college of cardiology; ESC European society of cardiology; JNC-8 eighth joint national committee; ASH American society of hypertension; SPRINT systolic blood pressure intervention trial; CHEP Canadian hypertension education program; ACP American college of physicians; AAFP American academy of family physicians)

treatment regimen. In a sub-group analysis of the 2636 participants aged 75 years or older [6], intensive treatment was associated to a reduction in primary composite endpoint of myocardial infarction, other acute coronary syndromes, stroke, heart failure, or cardiovascular death by $34 \%$ and all-cause mortality by $33 \%$ after a median followup of 3.14 years. At the same time, adverse events rate (hypotension, syncope, acute kidney injury, and electrolyte abnormalities was not significantly higher compared to the standard group. A positive aspect of this trial is represented by the large sample of elderly $\geq 75$ years ( $28.2 \%$ in each group) in study population. On the other hand, the exclusion of institutionalized adults and of subjects with a history of stroke, diabetes, or recent CVD symptoms, represents a strong limitation for SPRINT results application in clinical practice. In addition, the absence of attending physician, usually present during BP measurement in all other trial and in clinical practice, could have reduced the "white coat" effect that increase BP averages about $20 / 10 \mathrm{mmHg}$ after the doctor's/nurse's arrival [15], resulting in lower pressure values and making SPRINT data incomparable to other trials [16]. In the intensive treatment arm there was a greater use of diuretics, which may have masked the symptoms of heart failure rather than preventing them, and therefore, SPRINT results may have been unrelated to the difference BP reduction [17]. Mean SBP in intensive group was $123.4 \mathrm{mmHg}$, and consequently, a large part of elderly had a SBP out of target for the intensive treatment group $(<120 \mathrm{mmHg})$. In intensive arm more medications were needed (mean 2.8 vs. 1.8 ) to reach the SBP target although the use of multiple AHDs frequently lead to serious adverse events in elderly (i.e. orthostatic hypotension, dizziness, falls, syncope, and electrolyte abnormalities [18]. Finally, about $30 \%$ of intensive group patients were seen for unscheduled clinic visits, probably leading to a more accurate treatment in this arm and having more opportunity to report adverse effects [19].

\section{Guidelines post-SPRINT}

BP targets recommended for hypertension management in older adults are discordant in light of new evidence shown by SPRINT Study (Fig. 1). In 2017, The American college of physicians and the American academy of family physicians recommended a traditional approach in adults aged $>60$ years with a SBP goal $<150 \mathrm{mmHg}$ or $<140 \mathrm{mmHg}$ in patients with history of stroke or transient ischemic attack or with a high cardiovascular risk [20].

In the same year, Canadian guidelines for diagnosis, risk assessment, prevention, and treatment of hypertension in adults [21] did not recommend different approach for older adults, without a caution about frailty. The recent ACC/AHA hypertension guidelines for the prevention, detection, evaluation, and management of high blood pressure in adults recommend "SBP treatment goal of less than $130 \mathrm{mmHg}$ for non-institutionalized ambulatory community-dwelling adults ( $\geq 65$ years of age) with an average SBP of $130 \mathrm{~mm} \mathrm{Hg}$ or higher" (I-A recommendation), clearly based on SPRINT results.

In a lower degree recommendation (II-C), they suggest that "for older adults with high burden of comorbidity and limited life expectancy, clinical judgment, patient preference, and a team-based approach to assess risk/benefit is reasonable for decisions regarding intensity of BP lowering and choice of antihypertensive drugs". These recommendations seem to be far from a real-life elderly patient, often institutionalized and with high degree of comorbidity. [22] Thus, the therapeutic approach for these complex kind of patients is not clear and seems at least questionable as so strong recommendation based mainly on a single trial with a great deal of light and shade.

\section{Frailty and BP targets}

Frailty is considered as a geriatric dynamic condition characterized by an increased vulnerability to external stressors, by a multi-system dysfunction, by a complex etiology and by an intrinsic difficulty in distinguishing from aging [23]. Fried et al. defined frailty as a phenotype characterized by three or more of the following: unintentional weight loss of $10 \mathrm{lbs}$ or more in the past year, selfreported exhaustion, weakness measured by grip strength, slow walking speed, and low physical activity [24], describing only the physical domain of this condition. In contrast, Rockwood et al. consider frailty as a "clinical" 
condition and characterized by comorbidity, polypharmacy and relative adverse drug reactions, age-associated sensory deficits and lack of social support and high risk of disability, hospitalization, and health services utilization (multidimensional phenotype) [25].

Identifying older adults with a high frailty degree is extremely important in order to prevent aggressive drug regimens, to guarantee a safer approach and to improve quality of life [26]. There are few data on hypertension in the frail elderly. Post hoc analyses of SPRINT [6] and HYVET [27] trials suggest that frailty condition does not influence treatment effects on hypertensive subjects $[28,29]$. In fact, most trials in older adults did not assess frailty or exclude patients with moderate-severe degree of frailty.

In 2015, Ogliari et al. [30] investigated the relationship between BP and mortality in older adults according to age, functional and cognitive status. SBP higher than $165 \mathrm{mmHg}$ (but lower than $180 \mathrm{mmHg}$ ) and DBP higher than $85 \mathrm{mmHg}$ were associated with the lowest mortality in patients with impaired Activity daily living (ADL) and Mini mental state examination (MMSE) but not in those with preserved ADL and/or MMSE. These data prospect a personalized BP management in older adults using functional and cognitive status as markers of biological age [30]. A recent cohort study investigated the role of office and ambulatory blood pressure monitoring AHDs in predicting cognitive decline progression in patients with dementia or mild cognitive impairment (MCI) [31]. The authors showed an independent association between low daytime SBP and a greater progression of cognitive decline in older patients with dementia and mild cognitive impairment (MCI) among those treated with AHDs [31]. In 2016, a meta-analysis about the pressure treatment in older adults, collecting data from large trial including elderly [28], confirmed that a BP goal $<150 / 90 \mathrm{mmHg}$ improved outcomes such as mortality, CVD, stroke, CKD and cognitive decline. However, in this meta-analysis, the results supporting the positive effects of an intensive antihypertensive therapy in the elderly were largely deriving from SPRINT Trial [28].

In SPRINT Study thfe frailty status of participants was quantified using a 37-item frailty index (FI) and then subjects were classified as fit $(\mathrm{FI}<0.10)$, less fit $(0.10<\mathrm{FI}<$ $0.21)$, or frail $(\mathrm{FI}>0.21)$ [8]. In the exploratory sub-group analysis on subjects aged $>75$ years, a large part of population (30.9\% out of 2636) were classified as "frail". The authors indicated that benefits of intensive BP control were consistent among persons in this age range who were frail or had reduced gait speed [8]. Therefore, the study subgroup concluded that SPRINT Study "can successfully recruit a heterogeneous sample of non-institutionalized older adults" and that "these results also provide a future basis to evaluate the effect of lower BP targets on

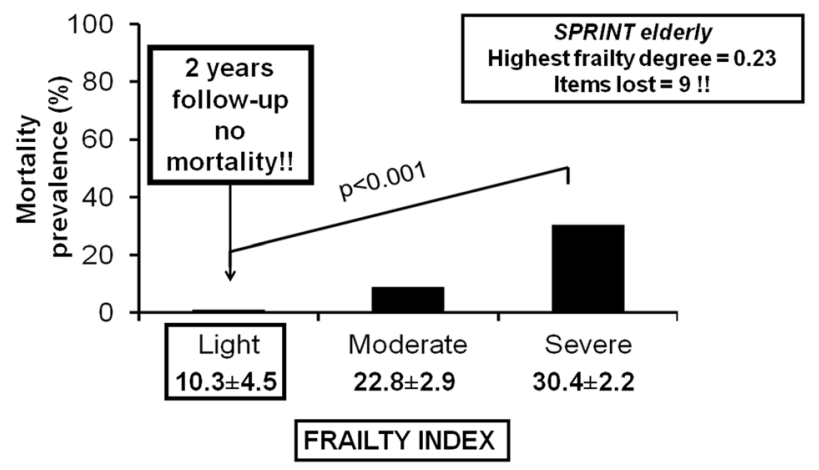

Fig. 2 Mortality rate in older adults stratified by Italian version of frailty index and its relation with frailty-related SPRINT analysis

cardiovascular events and mortality according to frailty status" [32].

However, these sentences appear questionable if we analyze the frailty degree of patients observed in SPRINT population. Frailty was defined by a FI $>0.21$ ( $>7$ items lost) and the median of FI in intensive treatment group was $0.18(0.13-0.23)$ that corresponds to a loss of $7( \pm 2)$ items and the median of FI in the standard treatment group was $0.17(0.12-0.22)$, a loss of $6( \pm 2)$ items. This means that the highest frailty degree observed in the sub-group study was equal to 0.23 , corresponding to a loss of $9 / 37$ items. In 2017, an Italian version of Frailty Index [33] (IFi) of 40 items was validated in a cohort of 1077 non-disabled outpatients aged 65 years or older $(81.3 \pm 6.5$ years). Frailty degree was stratified in light, moderate and severe according to IFi scores obtained. Mortality, disability ( $\geq 1$ ADL lost from the baseline) and hospitalization were considered at 3, 6, 12, 18 and 24 months of follow-up. In the light frailty group, the items lost were $10.3 \pm 4.5 / 40$ items and these participants had significant lower mortality rate compared to moderate and severe frailty group (Fig. 2). These subjects with a "light" frailty degree are similar to those considered "frail" by SPRINT study because of a loss of only 9 items. It is clear that SPRINT sub-study excluded moderate/severe frail elderly patients, making its conclusions nor applicable in the "real world". Thus, BP values associated with peculiar characteristics of severe frailty as the low body mass index and low serum total cholesterol, are clearly related to higher mortality in the elderly $[34,35]$.

\section{Conclusions}

The management of hypertension in the elderly is controversial. Several evidences suggest a SBP reduction not exceeding $140 \mathrm{mmHg}$ for its negative impact on elderly population. However, the SPRINT study seems to establish a positive effect of SBP reduction below $120 \mathrm{mmHg}$ even in subjects $\geq 75$ years old although several methodological 
problems make questionable the SPRINT results, including the exclusion of institutionalized adults, the automatic BP measuring in the absence of attending physician, the greater use of diuretics. In the elderly sub-study of SPRINT the most controversial issue is the selection of patients and the frailty degree evaluation that appears to be very light.

We conclude that SPRINT results can be applied only to general population but not in "frail" elderly patients. Specifically, frailty status should be not only identified but also well differentiated in different degree of frailty that might significantly influence the antihypertensive therapy in the elderly. Therefore, further prospective studies are required to define a safe BP target tailored specifically in different frailty degree.

Acknowledgements All authors contributed to the writing and editing of the manuscript and approved the final version submitted for publication. The manuscript has not been published or submitted elsewhere. No source of funds. No additional funding was obtained for this review.

\section{Compliance with ethical standards}

Conflict of interest The authors declare that they have no conflict of interest.

\section{References}

1. Bavishi C, Goel S, Messerli FH. Isolated systolic hypertension: an update after SPRINT. Am J Med. 2016;129:1251-8.

2. Hulley SB, Furberg CD, Gurland B, McDonald R, Perry HM, Schnaper HW, et al. Systolic hypertension in the elderly program (SHEP): antihypertensive efficacy of chlorthalidone. Am J Cardiol. 1985;56:913-20.

3. Staessen JA, Fagard R, Thijs L, Celis H, Arabidze GG, Birkenhäger WH, et al. Randomised double-blind comparison of placebo and active treatment for older patients with isolated systolic hypertension. The systolic hypertension in Europe (Syst-Eur) trial investigators. Lancet Lond Engl. 1997;350:757-64.

4. Beckett NS, Peters R, Fletcher AE, Staessen JA, Liu L, Dumitrascu D, et al. Treatment of hypertension in patients 80 years of age or older. N Engl J Med. 2008;358:1887-98.

5. SPRINT Research Group, Wright, Williamson JT, Whelton JD, Snyder PK, Sink JK. KM, et al. A randomized trial of intensive versus standard blood-pressure. Control $\mathrm{N}$ Engl J Med. 2015;373:2103-16.

6. Williamson JD, Supiano MA, Applegate WB, Berlowitz DR, Campbell RC, Chertow GM, et al. Intensive vs standard blood pressure control and cardiovascular disease outcomes in adults aged $\geq 75$ years: a randomized clinical trial. JAMA . 2016;315:2673-82.

7. JATOS Study Group. Principal results of the Japanese trial to assess optimal systolic blood pressure in elderly hypertensive patients (JATOS). Hypertens Res J Jpn Soc Hypertens. 2008;31:2115-27.

8. Ogihara T, Saruta T, Rakugi H, Matsuoka H, Shimamoto K, Shimada K, et al. Target blood pressure for treatment of isolated systolic hypertension in the elderly: valsartan in elderly isolated systolic hypertension study. Hypertens Dallas 1979. 2010; 56:196-202.
9. Benetos A, Labat C, Rossignol P, Fay R, Rolland Y, Valbusa F, et al. Treatment with multiple blood pressure medications, achieved blood pressure, and mortality in older nursing home residents: the PARTAGE Study. JAMA Intern Med. 2015;175:989-95.

10. Koh AS, Talaei M, Pan A, Wang R, Yuan J-M, Koh W-P. Systolic blood pressure and cardiovascular mortality in middle-aged and elderly adults-the Singapore Chinese health study. Int J Cardiol. 2016;219:404-9.

11. Mancia G, Fagard R, Narkiewicz K, Redón J, Zanchetti A, Böhm $\mathrm{M}$, et al. $2013 \mathrm{ESH} / \mathrm{ESC}$ guidelines for the management of arterial hypertension: the task force for the management of arterial hypertension of the European society of hypertension (ESH) and of the European society of cardiology (ESC). J Hypertens. 2013;31:1281-357.

12. James PA, Oparil S, Carter BL, Cushman WC, DennisonHimmelfarb C, Handler J, et al. 2014 Evidence-based guideline for the management of high blood pressure in adults: report from the panel members appointed to the eighth joint national committee (JNC 8). JAMA . 2014;311:507-20.

13. Weber MA, Schiffrin EL, White WB, Mann S, Lindholm LH, Kenerson JG, et al. Clinical practice guidelines for the management of hypertension in the community: a statement by the American society of hypertension and the international society of hypertension. J Clin Hypertens Greenwich Conn. 2014;16:14-26.

14. Rosendorff C, Lackland DT, Allison M, Aronow WS, Black HR, Blumenthal RS, et al. Treatment of hypertension in patients with coronary artery disease: a scientific statement from the American heart association, American college of cardiology, and American society of hypertension. Circulation. 2015;131:e435-70. CIR.0000000000000207

15. Celis H, Fagard RH. White-coat hypertension: a clinical review. Eur J Intern Med. 2004;15:348-57.

16. Kjeldsen SE, Lund-Johansen P, Nilsson PM, Mancia G. Unattended blood pressure measurements in the systolic blood pressure intervention trial: implications for entry and achieved blood pressure values compared with other trials. Hypertens Dallas 1979. 2016;67:808-12.

17. Kjeldsen SE, Narkiewicz K, Hedner T, Mancia G. The SPRINT study: Outcome may be driven by difference in diuretic treatment demasking heart failure and study design may support systolic blood pressure target below $140 \mathrm{mmHg}$ rather than below 120 mmHg. Blood Press. 2016;25:63-6.

18. Ricci F, De Caterina R, Fedorowski A. Orthostatic hypotension: epidemiology, prognosis, and treatment. J Am Coll Cardiol. 2015;66:848-60.

19. Cushman WC, Whelton PK, Fine LJ, Wright JT, Reboussin DM, Johnson KC, et al. SPRINT trial results: latest news in hypertension management. Hypertens Dallas 1979. 2016;67:263-5.

20. Qaseem A, Wilt TJ, Rich R, Humphrey LL, Frost J, Forciea MA, et al. Pharmacologic treatment of hypertension in adults aged 60 years or older to higher versus lower blood pressure targets: a clinical practice guideline from the American college of physicians and the American academy of family physicians. Ann Intern Med. 2017;166:430-7.

21. Leung AA, Daskalopoulou SS, Dasgupta K, McBrien K, Butalia S, Zarnke KB, et al. Hypertension Canada's 2017 guidelines for diagnosis, risk assessment, prevention, and treatment of hypertension in adults. Can J Cardiol. 2017;33:557-76.

22. Whelton PK, Carey RM, Aronow WS, Casey DE, Collins KJ, Dennison Himmelfarb C, et al. 2017 ACC/AHA/AAPA/ABC/ ACPM/AGS/APhA/ASH/ASPC/NMA/PCNA guideline for the prevention, detection, evaluation, and management of high blood pressure in adults. J Am Coll Cardiol. 2017. http://linkinghub. elsevier.com/retrieve/pii/S0735109717415191 Accessed 23 Jan 2018. 
23. Strandberg TE, Pitkälä KH. Frailty in elderly people. Lancet Lond Engl. 2007;369:1328-9.

24. Fried LP, Tangen CM, Walston J, Newman AB, Hirsch C, Gottdiener J, et al. Frailty in older adults: evidence for a phenotype. J Gerontol A Biol Sci Med Sci. 2001;56:M146-156.

25. Rockwood K, Mitnitski A. Frailty in relation to the accumulation of deficits. J Gerontol A Biol Sci Med Sci. 2007;62:722-7.

26. Muller M, Smulders YM, Leeuw PW, de, Stehouwer CDA. Treatment of hypertension in the oldest old: a critical role for frailty? Hypertension . 2014;63:433-41.

27. Warwick J, Falaschetti E, Rockwood K, Mitnitski A, Thijs L, Beckett N, et al. No evidence that frailty modifies the positive impact of antihypertensive treatment in very elderly people: an investigation of the impact of frailty upon treatment effect in the HYpertension in the Very Elderly Trial (HYVET) Study, a double-blind, placebo-controlled study of antihypertensives in people with hypertension aged 80 and over. BMC Med. 2015;13:78.

28. Weiss J, Freeman M, Low A, Fu R, Kerfoot A, Paynter R, et al. Benefits and harms of intensive blood pressure treatment in adults aged 60 years or older: a systematic review and meta-analysis. Ann Intern Med. 2017;166:419-29.

29. Peters R, Beckett N, McCormack T, Fagard R, Fletcher A, Bulpitt $\mathrm{C}$. Treating hypertension in the very elderly-benefits, risks, and future directions, a focus on the hypertension in the very elderly trial. Eur Heart J. 2014;35:1712-8.

30. Ogliari G, Westendorp RGJ, Muller M, Mari D, Torresani E, Felicetta I, et al. Blood pressure and 10-year mortality risk in the Milan Geriatrics 75+Cohort Study: role of functional and cognitive status. Age Ageing. 2015;44:932-7.

31. Mossello E, Pieraccioli M, Nesti N, Bulgaresi M, Lorenzi C, Caleri V, et al. Effects of low blood pressure in cognitively impaired elderly patients treated with antihypertensive drugs. JAMA Intern Med. 2015;175:578-85.

32. Pajewski NM, Williamson JD, Applegate WB, Berlowitz DR, Bolin LP, Chertow GM, et al. Characterizing frailty status in the systolic blood pressure intervention trial. J Gerontol A Biol Sci Med Sci. 2016;71:649-55.

33. Abete P, Basile C, Bulli G, Curcio F, Liguori I, Della-Morte D, et al. The Italian version of the "frailty index" based on deficits in health: a validation study. Aging Clin Exp Res. 2017;29:913-26.

34. Liguori I, Aran L, Bulli G, Russo G, Curcio F, Sasso G, et al. Statins in cardiovascular prevention in the oldest-old. A black hole. Off J Ital Soc Gerontol Geriatr. 2017;65:263-270.

35. Vischer UM, Safar ME, Safar H, Iaria P, Le Dudal K, Henry O, et al. Cardiometabolic determinants of mortality in a geriatric population: is there a "reverse metabolic syndrome"? Diabetes Metab. 2009;35:108-14. 